BULLETIN OF THE

AMERICAN MATHEMATICAL SOCIETY

Volume 77, Number 6, November 1971

\title{
CURVATURE AND COMPLEX ANALYSIS
}

\author{
BY R. E. GREENE ${ }^{1}$ AND H. WU ${ }^{2}$
}

Communicated by I. Singer, May 20, 1971

In this note, we announce some results in the geometric theory of several complex variables. For the first theorem, recall the theorem of Cartan-Hadamard: if $M$ is a Riemannian manifold with nonpositive Riemannian curvature, complete and simply connected, then it is diffeomorphic to Euclidean space. When the metric is actually Kähler, the following result gives additional information:

Theorem 1. Let $M$ be a complete simply connected Kähler manifold with nonpositive Riemannian curvature. Then

(i) $M$ is a Stein manifold.

(ii) If $\rho$ denotes the distance function from a fixed point $0 \in M$, then $\log \rho$ is plurisubharmonic and $\rho^{2}$ and $\log \left(1+\rho^{2}\right)$ are both $C^{\infty}$ and strictly plurisubharmonic. In fact

$$
d d^{c} \rho^{2} \geqq 4 \omega, \quad d d^{c} \log \left(1+\rho^{2}\right) \geqq 4 \omega /\left(1+\rho^{2}\right)^{2}
$$

where $d^{c}=(-1)^{1 / 2}\left(d^{\prime \prime}-d^{\prime}\right)$ and $\omega$ is the Kähler form of $M$.

(iii) If Riemannian curvature $\leqq-c^{2}<0$, then $d d^{c} \rho^{2} \geqq(2+2 c \rho \operatorname{coth} c \rho) \omega$, $d d^{c} \log \left(1+\rho^{2}\right) \geqq \alpha \omega$, where coth denotes the hyperbolic cotangent and $\alpha=\min \{2, c \operatorname{coth} c-1\}>0$.

(iv) If $-d^{2} \leqq$ Riemannian curvature $\leqq 0$, then

$$
\begin{aligned}
d d^{c} \rho^{2} & \leqq(4 \rho d \operatorname{coth} \rho d+2) \omega, \\
d d^{c} \log \left(1+\rho^{2}\right) & \leqq\left(1 /\left(1+\rho^{2}\right)\right)(4 \rho d \operatorname{coth} \rho d+2) \omega .
\end{aligned}
$$

Part (i) is a known result. See [4].

For the next theorem, we recall that it is generally conceded that no holomorphic function on $C^{n}$ can be in $L_{p}, p \leqq \infty$. In transferring this theorem to Kähler manifolds, it is obviously necessary to forego the case of $p=\infty$.

Theorem 2. Let $M$ be an $n$-dimensional complete simply connected Kähler manifold with nonpositive Riemannian curvature. Then

AMS 1969 subject classifications. Primary 5380, 5760, 3249; Secondary 3222, 3235.

${ }^{1}$ Supported partially by a Sloan Foundation Grant to the Courant Institute of Mathematical Sciences.

2 Sloan Fellow. Also supported partially by the National Science Foundation. Both authors gratefully acknowledge the generous advice and continued interest of P. A. Griffiths. 
(i) there exists no holomorphic function in $L_{p}, 0<p<\infty$. In fact, if $0 \in M$ is fixed, $S_{r}$ denotes the geodesic sphere of radius $r$ about 0 and $\omega_{r}$ denotes the volume form of the induced metric on $S_{r}$, then for every $f$ holomorphic in $M$,

$$
\int_{S_{r}}|f| p \omega_{r} \geqq|f(0)| p\left(\frac{2 \pi^{n}}{(n-1) !}\right) r^{2 n-1} .
$$

(ii) Suppose, in addition, Riemannian curvature $\leqq-c^{2}<0$, then

$$
\int_{S_{r}}|f|{ }^{p} \omega_{r} \geqq D_{f} e^{c r}
$$

for $r \geqq 1$ and for some constant $D_{f}$ independent of $r$.

The second part of this theorem may be used to show that Theorem 2.5.3 of Hörmander [2] cannot be generalized to Kähler manifolds of the type considered above. Theorem 2 is proved by first proving the corresponding statements for $C^{\infty}$ subharmonic functions on Riemannian manifolds. The following byproduct of the proof may be of independent interest: Let $M$ be a simply connected complete $d$-dimensional Riemannian manifold with nonpositive curvature and let $0 \in M$. Let $S_{r}$ be the geodesic sphere of radius $r$ around 0 and let $\omega_{r}$ be the volume form of the induced metric on $S_{r}$. If $f$ is a nonnegative $C^{\infty}$ subharmonic function on $M$, then

$$
f(0) \leqq \frac{1}{\sigma_{r}^{d-1}} \int_{S_{r}} f \omega_{r},
$$

where $\sigma_{r}^{d-1}$ denotes the volume of the sphere of radius $r$ in $R^{d}$. Suppose furthermore the metric of $M$ is spherically symmetric around 0 , then

$$
f(0)=\frac{1}{A_{r}} \int_{S_{r}} f \omega_{r}
$$

if $f$ is harmonic (where $A_{r}=$ volume of $S_{r}$ ), and

$$
f(0) \leqq \frac{1}{A_{r}} \int_{S_{r}} f \omega_{r}
$$

if $f$ is $C^{\infty}$ subharmonic (with no restriction on its sign).

Our third theorem complements Theorem 2 in that we exclude the possibility of any holomorphic function in $L_{\infty}$ under some restrictions on the curvature. Recall that the unit ball $B^{n}$ in $C^{n}$ admits bounded holomorphic functions and also that $B^{n}$ admits a complete Kähler 
metric with Riemannian curvature bounded from above by a negative constant (its Bergman metric).

THEOREM 3. Let $M$ be a complete simply connected Kähler manifold with nonpositive Riemannian curvature such that, for some $0 \in M$,

$$
\mid \text { Riemannian curvature }(p) \mid \leqq C(d(0, p))^{-3}
$$

for some constant $C$; then $M$ admits no bounded holomorphic function.

COROLlaRY. Let $M$ be a complete simply connected Kähler manifold with nonpositive Riemannian curvature which is flat outside a compact set. If $\eta$ is a holomorphic $n$-form on $M(n=\operatorname{dim} M)$, then $\int_{M} \eta \wedge \bar{\eta}=\infty$.

It is a known theorem (essentially due to Grauert and Reckziegel, see [5]) that $C^{n}$ cannot be given a Hermitian metric with holomorphic sectional curvature bounded from above by a negative constant. We shall improve on this result. Define a pseudo-Hermitian metric on $\mathbf{C}^{n}$ to be a Hermitian bilinear form $\sum_{\alpha, \beta} g_{\alpha \beta} d z^{\alpha} \otimes d \bar{z}^{\beta}$ such that (i) $\left(g_{\alpha \beta}\right)$ is $C^{\infty}$, (ii) $\left(g_{\alpha \beta}\right)$ is positive semidefinite, (iii) $\left(g_{\alpha \beta}\right)$ is positive definite outside a proper subvariety $S$ of $\boldsymbol{C}^{n}$.

TheORem 4. $C^{n}$ admits no pseudo-Hermitian metric with nonpositive Ricci curvature such that

$$
\|z\|^{2}(\text { Ricci curvature }(z)) \rightarrow-\infty \quad \text { as }\|z\| \rightarrow \infty .
$$

Corollary. For $n>1, C^{n}$ admits no pseudo-Hermitian metric such that

$$
\left.\|z\|^{2} \text { (holomorphic sectional curvature }(z)\right) \rightarrow-\infty \quad \text { as }\|z\| \rightarrow \infty .
$$

The Corollary is definitely false for $n=1$. In fact, one can easily construct a Hermitian metric on $\boldsymbol{C}$ which is complete and has Gaussian curvature -1 outside the unit disc.

We now state some properties of the Bergman kernel and Bergman metric of the geodesic balls of the Kähler manifolds of Theorem 1. We do so in a more general setting. Let $M$ be a Stein manifold and let $D$ be a bounded domain of $M$. The set of all holomorphic $n$-forms $\omega(n=\operatorname{dim} M)$ on $D$ such that $\int_{D} \omega \wedge \bar{\omega}<\infty$ is a Hilbert space. If $\left\{\omega_{i}\right\}$ is an orthonormal basis, we define the Bergman kernel form $\kappa$ in a standard manner

$$
\kappa=\sum_{i} \omega_{i} \wedge \bar{\omega}_{i}
$$

(compare Kobayashi [3]). Write locally $\kappa=K d z^{1} \wedge \cdots \wedge d \bar{z}^{n}$; then we define the Bergman metric $\beta$ to be 


$$
\beta=\sum_{i, j} \frac{\partial^{2} \log K}{\partial z^{i} \partial \bar{z}^{j}} d z^{i} \otimes d \bar{z}^{j} .
$$

Theorem 5. Let $M$ be a Stein manifold and let $D \subset \subset M$ be a strongly pseudo-convex domain of $M$ with $C^{2}$ boundary; then the Bergman kernel form goes to infinity at $\partial D$ and the Bergman metric of $D$ is complete.

This theorem was proved independently by N. Kerzman using the delicate estimates of Hörmander [2]. We are indebted to him for improvements in our proof. As a byproduct of this proof, we obtain

THEOREM $5^{\prime}$. Let $D \subset \subset C^{n}$ be a geometrically convex domain (with arbitrary boundary); then the Bergman kernel function goes to infinity at $\partial D$ and the Bergman metric is complete.

The analogue of Theorem 1 for positively curved Kähler manifolds is not known despite the many properties one now knows of such manifolds (see Gromoll-Meyer [1]). We have only a weak result in this direction.

THEOREM 6. Let $M$ be a noncompact complete Kähler manifold with positive Riemannian curvature; then $M$ has no compact complex submanifolds.

We actually prove the nonexistence of compact minimal submanifolds in such Riemannian manifolds.

Now we conclude this list of theorems with a sufficient condition for a domain in a Kähler manifold to be Stein. Recall that a convex body in $\mathbf{C}^{n}$ is Stein. The following result is of a similar nature. Let $D$ be a domain in a Kähler manifold $M$. We say $D$ is locally geodesically convex if and only if for each $p \in \partial D$, there is a neighborhood $U$ of $p$ such that for all $q$ in $U \subset D$, the shortest geodesic joining $p$ and $q$ is in $U \cap D$.

Theorem 7. Let $M$ be a Kähler manifold which is also Stein, and let $D$ be a domain in $M$ with $C^{2}$ boundary. If $D$ is locally geodesically convex, then $D$ is Stein.

We can give an interpretation of local geodesic convexity by stating the following extension of a theorem of Eberhard Schmidt: Let $M$ be a Riemannian manifold with nonpositive Riemannian curvature, simply connected and complete; then a domain $D \subset M$ with $C^{2}$ boundary is geodesically convex if and only if it is locally geodesically convex. Hence

Corollary. Let $M$ be a Kähler manifold with nonpositive Rieman- 
nian curvature, simply connected and complete; then every geodesically convex domain in $M$ with $C^{2}$ boundary is Stein.

\section{BIBLIOGRAPHY}

1. D. Gromoll and W. Meyer, On complete open manifolds of positive curvature, Ann. of Math (2) 90 (1969), 75-90. MR 40 \#854.

2. L. Hörmander, $L^{2}$ estimates and existence theorems for the $\bar{\partial}$ operator, Acta Math. 113 (1965), 89-152. MR 31 \#3691.

3. S. Kobayashi, Geometry of bounded domains, Trans. Amer. Math. Soc. 92 (1959), 267-290. MR 22 \#3017.

4. H. Wu, Negatively curved Kähler manifolds, Notices Amer. Math. Soc. 14 (1967), 515. Abstract \#67T-327.

5. - Normal families of holomorphic mappings, Acta Math. 119 (1967), 193-233. MR 37 \#468.

Courant Institute of Mathematical Sciences, New York University, New YORK, NEW YORK 10012

University of California, Berkeley, California 94720

Princeton University, Princeton, New Jersey 08540

Current address: (R. E. Greene) Department of Mathematics, University of California, Los Angeles, California 90024 